\title{
'A Prospective Case Control Study to Evaluate The CRADLE Vital Signs Alert Device For Detection of Patients With Clinically Important Malaria in Refugee Settings'.
}

Katy Kuhrt ( $\square$ katykuhrt24@gmail.com )

King's College London https://orcid.org/0000-0001-5016-0430

Paul T Seed

King's College London

Andrew H Shennan

King's College London

\section{Research}

Keywords: Malaria, community based surveillance, refugees, disease detection

Posted Date: February 18th, 2021

DOI: https://doi.org/10.21203/rs.3.rs-205157/v1

License: (-) (1) This work is licensed under a Creative Commons Attribution 4.0 International License. Read Full License 


\section{Abstract}

\section{Background}

Malaria is a significant threat to refugee populations. Bidibidi Refugee Settlement, Northern Uganda hosts 223000 of Uganda's 1.4 million refugees, vulnerable to malaria due to crowded conditions and limited access to preventative measures and health care. Early detection and referral of suspected malaria cases is key to reducing associated morbidity and mortality. We therefore aimed to evaluate shock index (heart rate/ systolic blood pressure), calculated by the CRADLE Vital Signs Alert (VSA) device, an easy-to-use blood pressure and heart rate monitor, for detection of malaria as grounds for whether the device could be used for low cost identification and referral of patients by non-medically trained Village Health Team workers (VHTs).

\section{Methods}

CRADLE VSA devices and related training were delivered to all health facilities and VHTs in Bidibidi Refugee Settlement from April to August 2018. CRADLE VSA readings was performed as part of routine patient assessment. CRADLE VSA data (blood pressure, heart rate) and assigned diagnoses were collected from health facility data record books and shock index calculated for each case. Cases were grouped into predefined disease categories, including malaria and severe malaria. A control group consisted of refugees undergoing asymptomatic screening using the CRADLE VSA. Average shock index was calculated for cases and controls and prespecified disease categories, and predictive statistics to evaluate shock index for prediction of malaria and severe malaria.

\section{Results}

Five hundred and eighty-seven CRADLE VSA devices were delivered. Malaria accounted for 26\% (915/3577) of cases and had the highest shock index compared to other disease categories. Positive likelihood ratios for shock index using a threshold of greater than or equal to 0.9 were 5 and 11 for malaria and severe malaria respectively.

\section{Conclusion}

Malaria accounted for over a quarter of cases. Positive likelihood ratios indicated that patients with shock index greater than or equal to 0.9 were 5 and 11 times more likely to be suffering from malaria and severe malaria respectively, indicating that the CRADLE VSA could be used by non-medically trained VHTs to identify patients likely to have malaria, and those most at risk of severe disease needing urgent referral.

\section{Trial Registration}

This is an observational study and therefore does not have or require a trial registration. Appropriate permissions were granted by UN Refugee Council, Ministry of Health and Office of the Prime Minister.

\section{Background}

Almost two-thirds of refugees live in malaria endemic regions. ${ }^{1}$ Uganda hosts 1.4 million refugees across 13 UN Refugee Agency (UNHCR) coordinated sites, including Bidibidi, the world's second largest refugee settlement, which spans $250 \mathrm{~km}^{2}$ and hosts 223000 refugees, mainly from South Sudan. ${ }^{2}$ Malaria is a leading cause of morbidity and mortality in Uganda accounting for 30 to $50 \%$ of outpatient visits to health facilities and up to $20 \%$ of deaths. ${ }^{3}$ Refugees are particularly vulnerable due to proximity of the settlements to thick vegetation and stagnant water (favourable breeding grounds for mosquitoes), lack of availability of preventative measures including mosquito nets and insecticide and poor access to healthcare. ${ }^{1}$ Early detection and treatment are key to reducing Malaria burden. ${ }^{4}$

However, whilst the use of rapid diagnostic tests (RDTs) was implemented in many refugee settlements, according to UNHCR health information system (HIS) data less than $45 \%$ of malaria cases are confirmed due to limited access to tests. ${ }^{1}$. Furthermore there is a $90 \%$ shortfall in the health work force to deliver basic health care, ${ }^{5}$ leading to an increasing reliance on non-medically trained community health workers (labelled as Volunteer Health Workers - VHTs), to perform community public health surveillance, including early identification of sick community members and prompt referral to health facilities for timely treatment. VHTs are currently poorly equipped for this role, in terms of training and equipment. 
The CRADLE Vital Signs Alert (VSA) is a cheap ( $£ 15 \mathrm{GBP}$ ), portable, easy-to-use tool which measures blood pressure (BP), heart rate (HR) and calculates shock index (SI) (heart rate/ systolic blood pressure) and was originally designed for use in pregnant women, but has also been shown to be accurate in non-pregnant adults. ${ }^{6,7,8}$ It incorporates a built in traffic light early warning system to alert the user to abnormal vital signs and the need for timely referral. The traffic lights are triggered by standard thresholds of blood pressure, as well as by shock index (heart rate/systolic blood pressure), a proven marker of early deterioration secondary to trauma in combat settings ${ }^{9,10}$ and obstetric haemorrhage and sepsis. ${ }^{11,12,13}$ For example, a 'red light' and 'up arrow' indicates severe hypertension and should prompt intervention and/or referral; a 'red light' and a 'down arrow' secondary to shock also requires urgent attention; a 'yellow light' and either a 'down arrow' or 'up arrow' indicates a need for a non-urgent assessment +/- referral. (Appendix 1).

The aim of this study was to evaluate shock index for detection of malaria and to establish whether the CRADLE VSA could enable low cost identification of patients at risk of severe malaria by VHTs, enabling prompt diagnosis and treatment.

\section{Methods}

\section{Design and setting}

This was a prospective case control study to evaluate the CRADLE VSA for detection of patients with clinically important malaria (defined by local staff) in Bidibidi Refugee Settlement, Yumbe district, Northern Uganda.

\section{Participants}

All symptomatic patients aged 18 years or above presenting to a healthworker (VHT in the community or a health worker based at a health facility), were eligible to participate.

\section{Processes/ data collection}

One hundred and thirty six CRADLE VSAs were distributed to each of the 17 UNHCR health facilities (8 devices per health facility), and VHTs received their own device, between April to August 2018. This was supported by the CRADLE training package, delivered to all health workers (health facility based and VHTs) including one off face-to-face training by the research team lasting approximately 5 hours (presentation, short animated training films), and training materials: printed booklets and aide memoire cards attached to each device. All participants had a CRADLE VSA reading performed as part of their routine assessment. If the results showed red or yellow the reading was repeated. If the yellow or red showed only once a third measurement was taken and the most consistent light was followed in terms to inform referral.

Data was routinely collected (CRADLE VSA measurement including systolic blood pressure (SBP), diastolic blood pressure (DBP) and pulse rate (PR) and diagnosis (designated by the attending health facility worker)) from data record books at each health facility. Shock index was calculated for each patient (pulse rate/ systolic blood pressure).

All cases were reviewed and grouped into the following predefined disease categories, blinded to the CRADLE VSA measurements: anaemia/ fatigue; bacteraemia/ febrile illness; cardiovascular; dermatology; eye/ ENT; gastrointestinal/ diarrhoeal illness; genitourinary; malaria; musculoskeletal; neurology/psychiatry; respiratory; other. Malaria diagnoses were assigned after a positive rapid diagnostic test (as per health facility standard clinical practice). Additional analysis was performed on malaria cases defined as 'severe' by local clinical staff.

A control group consisted of refugees undergoing asymptomatic screening using the CRADLE VSA in surrounding villages within the settlement.

\section{Statistics}

Power calculation was based on the assumption of a $10 \%$ prevalence of malaria, and a sensitivity and specificity of $60 \%$ for shock index (positive likelihood ratio 1.5). We calculated that 660 subjects would be needed ( 60 subjects with a disease falling into one of the eleven disease categories of interest) for $90 \%$ power. 
Mean shock index was calculated for cases and controls, and for each disease category. Predictive statistics, including positive and negative likelihood ratio (LR+/LR-), odds ratio and ROC area, were calculated to evaluate shock index for prediction of malaria and severe malaria. Positive and negative predictive values were not included as no reliable prevalence figures were available for severe malaria.

\section{Results}

All of the CRADLE VSA devices were successfully delivered to the health facilities and 451 VHTs received their own device. Data was collected on 3577 symptomatic cases (76\% female; $24 \%$ male) and 2163 asymptomatic controls (67\% female; $33 \%$ male) (Table 1) between April to August 2018.

Table 1. Demographic table to show characteristics of Cases and controls.

\begin{tabular}{|llll|}
\hline Characteristic & Control, $\mathbf{n}(\%)$ & Cases, $\mathbf{n}(\%)$ & Both Groups, $\mathbf{n}(\%)$ \\
\hline $\mathbf{n}$ & 2163 & 3577 & 5740 \\
\hline Mean Age & 33 & 36 & 35 \\
\hline Female & $1453(67.2)$ & $2717(76.1)$ & $4170(72.7)$ \\
\hline Male & $710(32.8)$ & $852(23.9)$ & $1562(27.3)$ \\
\hline Pregnant & $160(7.4)$ & $125(3.5)$ & $285(5.0)$ \\
\hline CRADLE VSA traffic light & & \\
\hline Green & $1688(78)$ & $2530(70.7)$ & $4218(73.5)$ \\
\hline Yellow Up & $312(14.4)$ & $479(13.4)$ & $791(13.8)$ \\
\hline Yellow Down & $70(3.2)$ & $359(10.0)$ & $429(7.5)$ \\
\hline Red Up & $93(4.3)$ & $209(5.8)$ & $302(5.3)$ \\
\hline
\end{tabular}

Mean shock index ( $\mathrm{SI}$ ) was 0.68 (95\% confidence interval 0.67 to 068$)$ and 0.64 (95\% confidence interval 0.63 to 0.65$)$ in cases and controls respectively which was significantly different $(0.039,95 \% \mathrm{Cl} 0.03$ to 0.0047$)$. Malaria accounted for $26 \%(915 / 3577)$ of the cases, had the highest SI compared to other disease categories $(0.72,95 \% \mathrm{Cl} 0.26$ to 1.67$)$ (figure 1$)$, and the greatest proportion of shock index greater than 0.9 (15\%) compared to other disease categories. (figure 2)

Predictive statistics for all malaria cases and severe malaria only, based on a shock index threshold of 0.9 are shown in table 2 .

Table 2. Predictive statistics for all malaria cases vs severe malaria only.

\begin{tabular}{|lll|}
\hline Using a threshold of SI greater than or equal to 0.9 for a positive test. & \multicolumn{2}{l|}{ (95\% Confidence Interval, $\mathbf{p}<0.05)$} \\
\hline $\mathrm{n}$ & All Malaria & Severe Malaria \\
\hline Positive likelihood ratio & 929 & 38 \\
\hline Negative likelihood ratio & $4.6(3.5$ to 6.10$)$ & $11.4(7.1$ to 18.3$)$ \\
\hline Odds Ratio & $0.88(0.85$ to 0.90$)$ & $0.65(0.51$ to 0.83$)$ \\
\hline ROC area & $5.3(3.9$ to 7.2$)$ & $17.44(8.0$ to 36.7$)$ \\
\hline
\end{tabular}

\section{Discussion}

Malaria accounted for over a quarter of cases in our cohort. Positive likelihood ratios indicated that patients with a shock index greater than or equal to 0.9 were 5 and 11 times more likely to be suffering from malaria and severe malaria respectively, indicating that the CRADLE VSA could be used to identify patients likely to have malaria, and to determine those most at risk of severe disease. 
The key to prevention of significant morbidity and mortality related to malaria is early detection. Although false negatives and positives are common with moderate ROC areas (table 2) meaning that the CRADLE VSA does not represent a reliable diagnostic test, it has potential clinical utility as a community based screening tool for early identification of those in need of referral by non-medically trained VHTs. This is well aligned with the Ugandan Ministry of Health growing emphasis on rapidly expanding and equipping health teams through VHTs to carry out community based surveillance including rapid detection and referral of suspected malaria cases and other life threatening conditions.

Previous work in India, Mozambique and South Africa has shown that unskilled community health workers perceive the CRADLE VSA as easy-to-use ${ }^{14}$ and it meets the specific WHO requirements for use in low income settings, being affordable (£15 GBP), robust, portable with low power requirements. ${ }^{15}$

\section{Limitations}

Accurate diagnoses of conditions were prone to misclarification, and although malaria cases were determined by RDTs, those cases defined as 'severe' were based on clinicians' judgement. Pragmatic definitions were used in this challenging low resource setting to establish clinical utility of the CRADLE VSA in this context.

\section{Conclusion}

Cheap, easy-to-use tools up-skilling VHTS and other health workers performing community based surveillance in similar settings could help to identify patients likely to be suffering from malaria, which remains a significant threat to the health of refugee populations, especially in Sub-Saharan Africa. ${ }^{1}$. Further work in screening other refugee populations is needed.

\section{Declarations}

\section{Ethical approval and consent to participate:}

This is an observational study and therefore does not have or require a trial registration or ethical approval. Blood pressure measurements were taken and recorded as part of routine clinical care, therefore participants were not consented. Appropriate permissions were granted by UN Refugee Council and Ministry of Health.

\section{Consent for publication:}

Not Applicable.

\section{Availability of data and materials:}

The datasets analysed during the current study are available from the corresponding author on reasonable request.

\section{Competing interests:}

The authors declare that they have no competing interests.

\section{Funding:}

This study was funded by MRC [MR/N006240/1]. Funding was used to purchase CRADLE VSA devices and to support health worker training and associated costs (venue hire, subsistence, stationary).

\section{Authors' contributions:}

KK collected, analysed and interpreted participant data and wrote the manuscript. PS performed statistical analysis on the data; AS analysed and interpreted participant data and was a major contributor in writing the manuscript. All authors read and approved the final manuscript.

\section{Acknowledgements:}


We thank Sharon Charles Nabwira (local project coordinator), Emma Cordell, Michael Shennan and Léa Adamson who assisted with organisation and delivery of training and data collection.

\section{References}

1. Anderson J, Doocy S, Haskew C, Spiegel P, Moss WJ. The burden of malaria in post-emergency refugee sites: A retrospective study. Confl Health. 2011;5(1):17 doi:10.1186/1752-1505-5-17

2. The UN Refugee Agency. Coronavirus Outbreak. [Accessed on 12 May 2020 at https://www.unhcr.org/uk/coronavirus-covid19.html]

3. National Malaria Control Program. Ministry of Health, republic of Uganda. [Accessed on $9^{\text {th }}$ June 2020 at https://www.health.go.ug/programs/national-malaria-control-program/]

4. The Republic of Uganda Ministry of Health. Press Statement on the Mosquito Net distribution among Refugees in Uganda, $20^{\text {th }}$ June 2018. [Accessed on 03/09/2018 at go.ug/download/file/fid/1907]

5. The Republic of Uganda Ministry of Health. Operational Guidelines on COVID-19 for Village Health Teams (VHTs).

6. Nathan HL, de Greeff A, Hezelgrave NL, Chappell LC, Shennan AH. An accurate semi- automated oscillometric blood pressure device for use in pregnancy (including pre-eclampsia) in a low-income and middle-income country population: the Microlife 3AS12. Blood Press Monit 20:52- 55, 2015 Wolters Kluwer Health, Inc.

7. Nathan HL, de Greeff A, Hezelgrave NL, Chappell LC, Shennan AH. Accuracy validation of the Microlife 3AS1-2 blood pressure device in a pregnant population with low blood pressure. Blood Press Monit Wolters Kluwer Health, Inc.

8. de Greeff A, Nathan H, Stafford N, Liu B, Shennan AH. Development of an accurate oscillometric blood pressure device for low resource settings. Blood Press. Monit. 2008;13(6):342-8.

9. Cannon $\mathrm{CM}$, Braxton $\mathrm{CC}$, Kling-Smith M, et al. Utility of the shock index in predicting mortality in traumatically injured patients. J Trauma 2009;67:1426-30.

10. Sloan EP, Koenigsberg M, Clark JM, et al. Shock index and prediction of traumatic hemorrhagic shock 28-day mortality: data from the DCLHb resuscitation clinical trials. West J Emerg Med 2014;15:795.

11. El Ayadi AM, Nathan HL, Seed PT, et al. Vital sign prediction of adverse maternal outcomes in women with hypovolemic shock: the role of shock index. PLoS One 2016;11:e0148729.

12. Nathan HL, Cottam K, Hezelgrave NL, et al. Determination of normal ranges of shock index and other haemodynamic variables in the immediate postpartum period: a cohort study. PLoS One 2016;11:e0168535.

13. Nathan HL, El Ayadi A, Hezelgrave NL, et al. Shock index: an effective predictor of outcome in postpartum haemorrhage? BJOG 2015;122:268-75.

14. Nathan HL, Boene H, Munguambe K, Sevene E, Akeju D, Adetoro 00 et al. The CRADLE vital signs alert: qualitative evaluation of a novel device designed for use in pregnancy by healthcare workers in low-resource settings. Reproductive Health (2018) 15:5 DOI 10.1186/s12978-017-0450-y

15. World Health Organisation Affordable Technology: Blood Pressure Measuring devices for Low Resource Settings, 2005. [Accessed on $03 / 09 / 2018$ at http://apps.who.int/iris/bitstream/handle/10665/43115/9241592648.pdf;jsessionid=BB842AC415ECEA4625ACBCB948CBFEF4? sequence=1]

\section{Figures}




\section{Mean Shock Index by Disease Category}

0.75
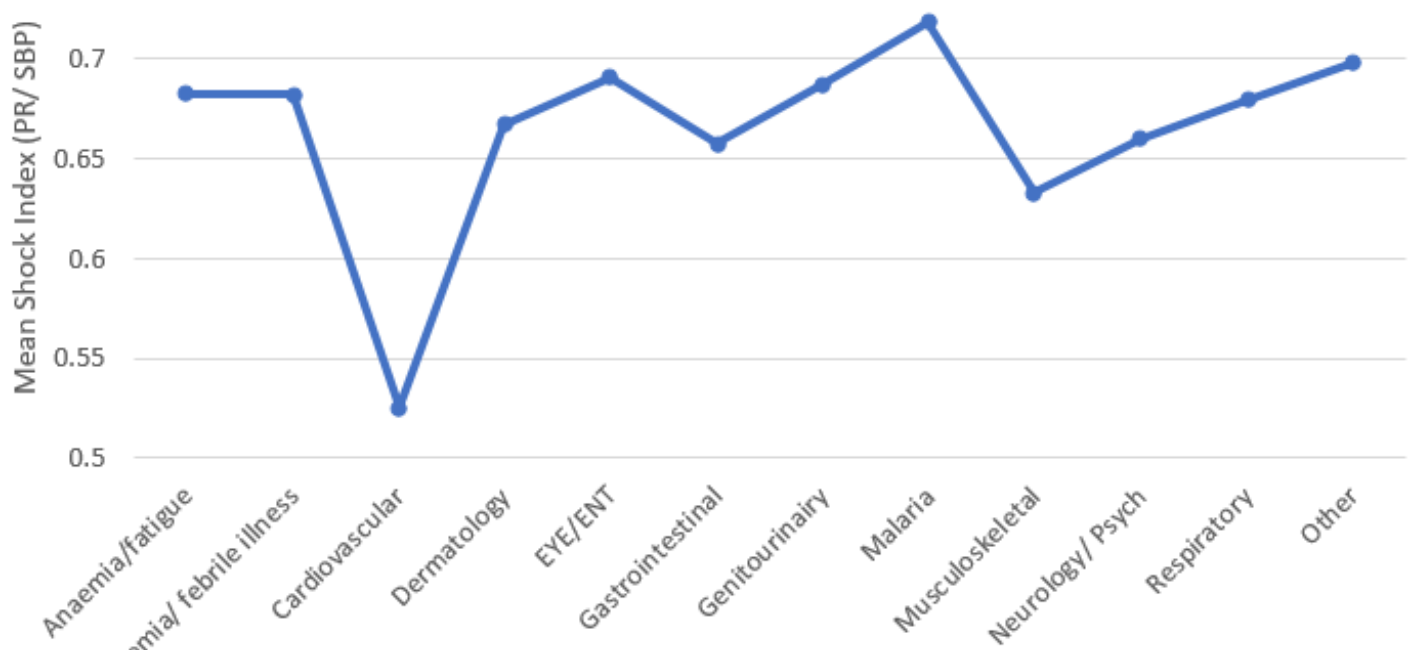

Primary Diagnosis Disease Category

\section{Figure 1}

Mean Shock Index by Disease Category.

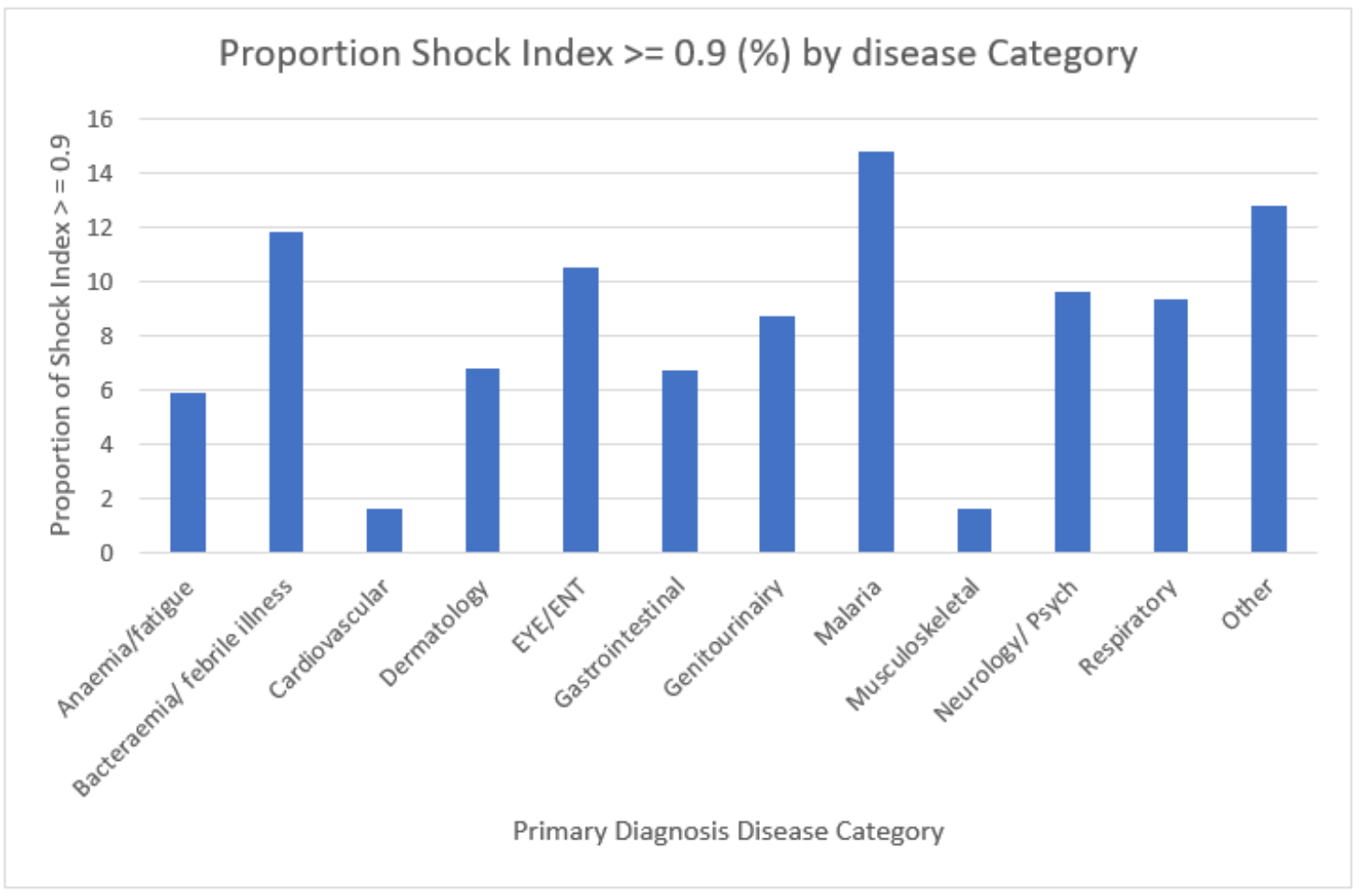

Figure 2

Proportion of Shock Index greater than or equal to 0.9 by disease category.

\section{Supplementary Files}


This is a list of supplementary files associated with this preprint. Click to download.

- Appendix1.docx 